\title{
Tumor neuroectodérmico primitivo periférico primário da órbita: relato de caso
}

\author{
Primary peripheral primitive neuroectodermal tumour of the orbit: case report
}

\author{
Ivana Lopes Romero ${ }^{1}$ \\ Luciano Sousa Pereira ${ }^{2}$ \\ Felipe Augusto Garcez de Campos $^{3}$ \\ José Vital Filho ${ }^{4}$ \\ Simone Haber Duellberg' Von Faber Bison ${ }^{5}$
}

\begin{tabular}{|c|}
\hline RESUMO \\
\hline Os autores relatam o primeiro caso de tumor neuroectodérmico primitivo \\
periférico primário da órbita apresentado na literatura nacional. Caracte- \\
rísticas clínicas, radiológicas e histopatológicas são discutidas. O diag- \\
nóstico foi confirmado por meio de análise imuno-histoquímica, etapa \\
essencial no diagnóstico dos tumores de pequenas células redondas.
\end{tabular}

Descritores: Tumores neuroectodérmicos/diagnóstico; Órbita/patologia; Imuno-histoquímica; Sarcoma de Ewing; Relatos de casos [Tipo de publicação]

\section{INTRODUÇÃO}

Tumor neuroectodérmico primitivo (PNET) é um termo utilizado para descrever uma categoria de tumores de pequenas células redondas com alto potencial de malignidade, cuja origem está relacionada às células da crista neural. A maioria dos PNETs ocorrem no sistema nervoso central ${ }^{(1)}$.

Os tumores neuroectodérmicos primitivos periféricos (pPNETs) são tumores malignos de partes moles que estão localizados fora do sistema nervoso central e sistema nervoso simpático. Representam 4 a $17 \%$ dos tumores de partes moles na infância ${ }^{(2)}$ e têm sido relatados em diferentes locais ${ }^{(3-4)}$.

O pPNET primário da órbita é extremamente raro e em nosso conhecimento somente 10 casos foram publicados previamente, nenhum deles em literatura nacional ${ }^{(5-9)}$. Neste estudo, apresentamos um caso de pPNET de órbita em uma criança de 10 meses de idade, com ênfase em suas características histopatológicas e imuno-histoquímicas.

Acadêmico do 4ํano da Faculdade de Ciências Médicas da Santa Casa de São Paulo (SP) - Brasil.

${ }^{4}$ Chefe do Setor de Órbita do Departamento de Oftalmologia da Santa Casa de São Paulo - São Paulo (SP) Brasil.

${ }^{5}$ Doutora, Professora e Chefe do Setor de Vias Lacrimais do Departamento de Oftalmologia da Santa Casa de São Paulo - São Paulo (SP) - Brasil.

Endereço para correspondência: Ivana Lopes Romero. Rua Martinico Prado, 284 - Apto. 54 - São Paulo (SP) CEP 01224-010

E-mail: ilromero@gmail.com

Recebido para publicação em 19.02.2008

Última versão recebida em 22.07.2008

Aprovação em 11.08.2008

Nota Editorial: Depois de concluída a análise do artigo sob sigilo editorial e com a anuência da Dra. Martha Maria Motono Chojniak sobre a divulgação de seu nome como revisora, agradecemos sua participação neste processo.

\section{RELATO DO CASO}

Paciente de 10 meses de idade, feminina, sem antecedentes pessoais ou familiares significantes, procurou o serviço de Órbita da Santa Casa de São Paulo em julho de 2006, apresentando tumoração na órbita direita de crescimento rápido ao longo de um mês. Ao exame oftalmológico, constatou-se apenas deslocamento súpero-temporal do globo ocular, ocasionado por lesão expansiva bem delimitada e indolor à palpação.

A tomografia computadorizada de órbita mostrou massa extraconal bem delimitada de aspecto ligeiramente heterogêneo e sem invasão de estruturas vizinhas com moderada captação de contraste (Figura 1).

O tumor foi ressecado sob anestesia geral e encaminhado para exame anátomo-patológico. O estudo histopatológico revelou presença de neoplasia neuroectodérmica indiferenciada de pequenas células redondas, que em diversos campos assumiam disposição de rosetas (Figura 2A). 
A pesquisa imuno-histoquímica foi positiva para AE1, AE3, proteína S100 (Figura 2B), CD 99, enolase, vimentina, WT-1, EMA (antígeno epitelial de membrana) e desmina, caracterizando o tumor como neuroectodérmico primitivo.

A avaliação sistêmica através de hemograma, mielograma, ultra-sonografia abdominal e pélvica foi negativa o que levou ao diagnóstico definitivo de pPNET primário da órbita. A paciente foi tratada de acordo com o protocolo dos setores de hematologia e oncologia pediátrica da Irmandade da Santa Casa de Misericórdia de São Paulo para sarcomas de alto grau, sendo submetida a 18 ciclos de quimioterapia e 5 sessões de radioterapia nas semanas de números 12 a 16, numeradas a partir do início de tratamento em agosto de 2006 (Tabela 1).

Após 17 meses de cirurgia e 18 ciclos de quimioterapia, além das sessões de radioterapia, não há sinais de recorrência.

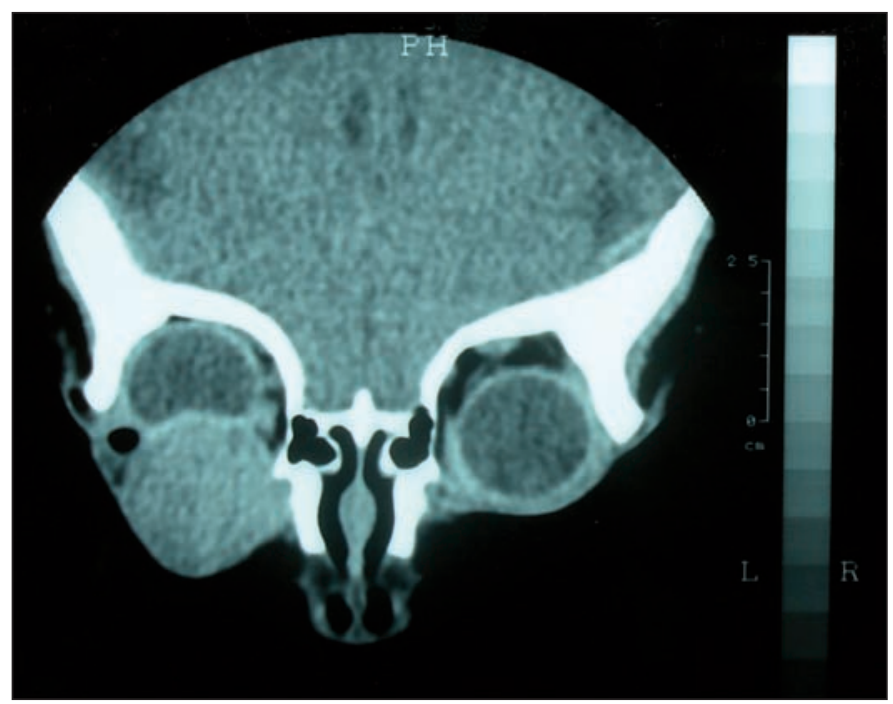

Figura 1 - Tomografia computadorizada de órbita, corte coronal, evidenciando presença de massa bem delimitada e heterogênea na órbita direita com deslocamento súpero-temporal do globo ocular

\section{DISCUSSÃO}

O pico de incidência dos pPNETs é na adolescência e não tem predileção por sexo ${ }^{(9)}$, no entanto quando avaliamos os 10 relatos de pPNET orbitários na literatura, notamos que em 7 deles o tumor ocorreu na infância e em apenas um, de forma semelhante ao nosso caso, o tumor foi diagnosticado no primeiro ano de vida ${ }^{(5-6,9)}$.

O diagnóstico diferencial do pPNET orbitário deve ser feito com outros tumores de pequenas células redondas incluindo tumor de Ewing extra-ósseo, neuroblastoma, rabdomiossarcoma, linfoma, sarcoma osteogênico e condrossarcoma mesenquimal.

Antigamente, os pPNETs faziam parte do grupo dos tumores de Ewing extra-ósseos, com os quais compartilhavam alterações microscópicas genéticas e moleculares, por exemplo a translocação $\mathrm{t}(11 ; 22)(\mathrm{q} 24 ; \mathrm{q} 12)^{(10)}$. Muitos autores consideram que os pPNETs e os tumores de Ewing extra-ósseos representam extremos de um mesmo grupo de doenças. Portanto, as técnicas de imuno-histoquímica e ultra-estruturais são fundamentais para estabelecer o diagnóstico diferencial entre esses tumores.

Histologicamente, os pPNETs apresentam um padrão monótono de alta celularidade, sendo formado por pequenas células redondas com núcleo hipercromático e variados graus de diferenciação neuronal. O processo de diferenciação é progressivo e inicia-se com a expressão de uma enolase específica neuronal, seguido pela formação de rosetas de HomerWright, diferenciação em células ganglionares e finalmente, pela expressão de proteínas de neurofilamento ${ }^{(11)}$. A presença de células assumindo disposição em rosetas representa evidência de que a lesão aqui estudada passou por esse processo de diferenciação neuronal. No entanto, esse achado isoladamente não é suficiente para o diagnóstico definitivo.

Os pPNETs apresentam na imuno-histoquímica positividade para: vimentina, CD19, glicoproteína p30-p32, além dos marcadores neuronais (neurofilamentos, sinaptofisina, cromogranina e enolase neuronal específica) ${ }^{(2)}$. Quanto à ultra-
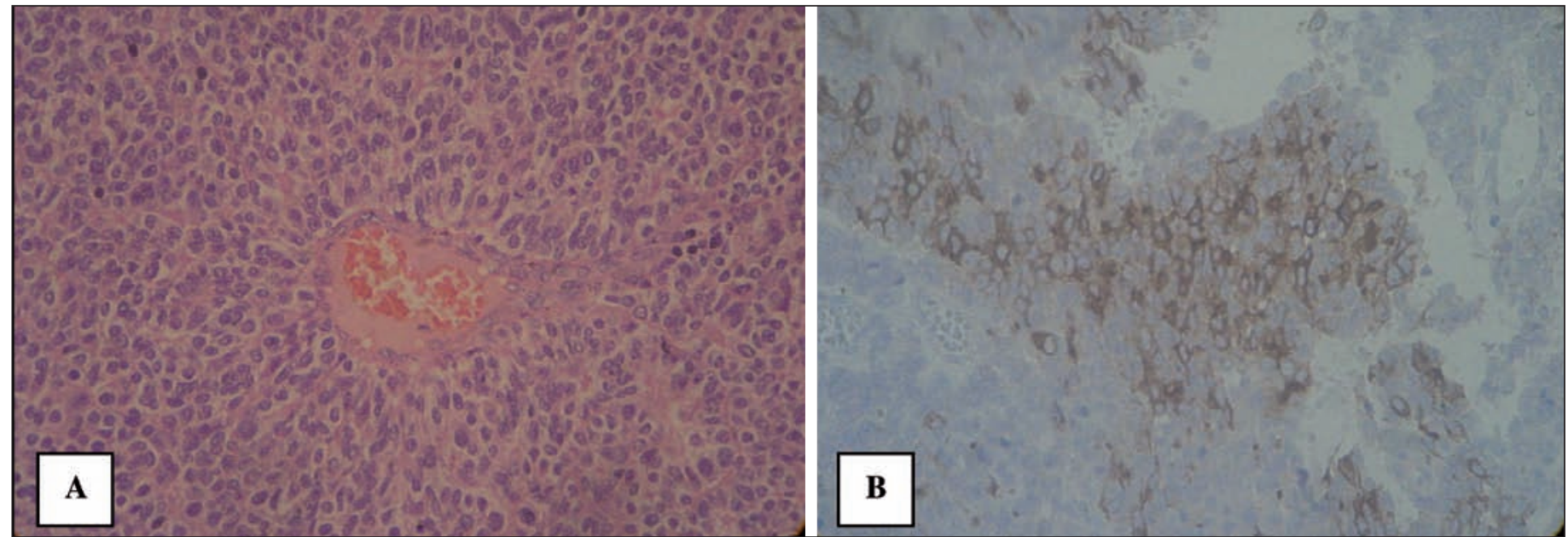

Figura 2 - A) Aspecto histopatológico de neoplasia neuroectodérmica indiferenciada de pequenas células redondas, onde se observa presença de roseta. B) Proteína S100 positivo. 


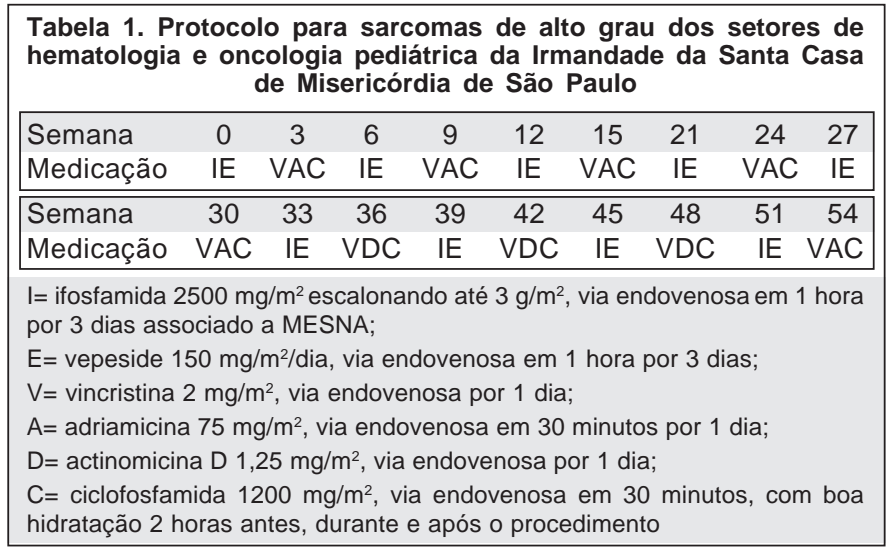

estrutura, podemos encontrar filamentos citoplasmáticos intermédios, microtúbulos e grânulos secretores.

Os pPNETs são tumores que progridem rapidamente e geralmente, apresentam mau prognóstico ${ }^{(2)}$. Quando localizados na órbita podem comprometer os ossos locais, metástases sistêmicas, entretanto, são raras, havendo 2 casos descritos na literatu$\mathrm{ra}^{(2,12)}$ cujos sítios foram pulmão e fígado. De forma semelhante, as recidivas não são freqüentes tendo ocorrido em $3 \operatorname{casos}^{(2,5,8)}$.

Não há consenso na literatura quanto ao tratamento. Com algumas exceções ${ }^{(9,13)}$, os pacientes foram submetidos a procedimento cirúrgico (biópsia excisional) associado a tratamento complementar com quimioterapia e radioterapia segundo protocolos específicos ${ }^{(2,6-8,14)}$, fato que também foi observado em nosso caso.

Concluindo, descrevemos um caso raro de pPNET orbitário acometendo um lactente de 10 meses de idade. As características imuno-histoquímicas aqui descritas são essenciais para o diagnóstico acurado dessas lesões.

\section{ABSTRACT}

The authors present a case of primary peripheral primitive neuroectodermal tumor of the orbit in a 10-month-old girl, which is the first case in the Brazilian literature. Clinical, radiologic and histopathologic features are discussed. The diagnosis was confirmed by immunohistochemical analysis which is essential to the diagnosis of small round cell tumors in the orbit.

Keywords: Neuroectodermal tumors/diagnosis; Orbit/pathology; Small round cell; Immunohistochemistry; Sarcoma; Ewing's; Case reports [Publication Type]

\section{REFERÊNCIAS}

1. Dehner LP. Primitive neuroectodermal tumor and Ewing's sarcoma. Am J Surg Pathol. 1993;17(1):1-13. Review.

2. Romero R, Abelairas J, Sanz J, Ruiz MM, Sendagorta E. [Recurrence of peripheral primitive neuroectodermal tumor of the orbit with systemic metastases]. Arch Soc Esp Oftalmol. 2006;81(10):599-602. Spanish.

3. Thete N, Rastogi D, Arya S, Singh A, Rao P, Chandge A, Ramadwar M. Primitive neuroectodermal tumour of the prostate gland: ultrasound and MRI findings. Br J Radiol. 2007;80(956):e180-3.

4. Blattner JM, Gable P, Quigley MM, McHale MT. Primitive neuroectodermal tumor of the uterus. Gynecol Oncol. 2007;106(2):419-22.

5. Howard GM. Neuroepithelioma of the orbit. Am J Ophthalmol. 1965;59:934-7.

6. Singh AD, Husson M, Shields CL, De Potter P, Shields JA. Primitive neuroectodermal tumor of the orbit. Arch Ophthalmol. 1994;112(2):217-21.

7. Kiratli H, Bilgic S, Gedikoglu G, Ruacan S, Ozmert E. Primitive neuroectodermal tumor of the orbit in an adult. A case report and literature review. Ophthalmology. 1999;106(1):98-102.

8. Lezrek M, Skiker H, Tachfouti S, Karim A, Karmane A, Bencherif Z, et al [Orbital primitive neuroectodermal tumor with intracranial extension. A case report]. J Fr Ophtalmol. 2005;28(9):982. French.

9. Tamer C, Oksuz H, Hakverdi S, Karazincir S, Balci A, Yaldiz M. Primary peripheral primitive neuroectodermal tumour of the orbit. Can J Ophthalmol. 2007;42(1):138-40.

10. Fletcher JA, Kozakewich HP, Hoffer FA, Lage JM, Weidner N, Tepper R, et al. Diagnostic relevance of clonal cytogenetic aberrations in malignant softtissue tumors. N Engl J Med. 1991;324(7):436-42.

11. Kleinert R. Immunohistochemical characterization of primitive neuroectodermal tumors and their possible relationship to the stepwise ontogenetic development of the central nervous system. 2. Tumor studies. Acta Neuropathol. 1991;82(6):508-15.

12. Hyun CB, Lee YR, Bemiller TA. Metastatic peripheral primitive neuroectodermal tumor (PNET) masquerading as liver abscess: a case report of liver metastasis in orbital PNET. J Clin Gastroenterol. 2002;35(1):93-7.

13. Bansal RK, Gupta A. Primitive neuroectodermal tumour of the orbit: a case report. Indian J Ophthalmol. 1995;43(1):29-31.

14. Wilson WB, Roloff J, Wilson HL. Primary peripheral neuroepithelioma of the orbit with intracranial extension. Cancer. 1988;62(12):2595-601. 